\title{
Challenges in promoting clinical empathy skills in medical students: A content analysis study
}

\author{
Nahid Ahmadian Yazdi ${ }^{1}$, Kamran Soltani Arabshahi ${ }^{1,2}$, Shoaleh Bigdeli*2, Saeideh Ghaffarifar ${ }^{3}$ \\ Received: 15 Apr 2018 \\ Published: 1 Oct 2019
}

\section{Abstract}

Background: Empathy is a key clinical skill in the medical profession, and many studies have reported a decline in it among medical students during their years of education; especially, in the clinical stage, and this affective decline persists in the physicianhood. This study aimed to explore the participants' perceptions about challenges for promoting clinical empathy in training stages.

Methods: A qualitative design using content analysis was applied. Semi-structured interviews were applied to obtain data. Individual interviews were conducted with 14 interns and six clinical professors. The data were analyzed through conventional content analysis and the credibility, trustworthiness, and conformability of the data were confirmed.

Results: Data analysis led to the extraction of two main categories, including overt and covert challenges, and also four categories and nine sub-categories.

Conclusion: Resolving clinical empathy challenges in medical students requires financial and human resources, and training on the principles of effective doctor-patient interactions. Furthermore, professionalism should be strengthened in professors, and both official and hidden curricula should be revised accordingly.

Keywords: Medical students, Qualitative study, Empathy, Challenge

Conflicts of Interest: None declared

Funding: Iran University of Medical Sciences

\section{*This work has been published under CC BY-NC-SA 1.0 license. \\ Copyright $\odot$ Iran University of Medical Sciences}

Cite this article as: Ahmadian Yazdi N, Soltani Arabshahi K, Bigdeli Sh, Ghaffarifar S. Challenges in promoting clinical empathy skills in medical students: A content analysis study. Med J Islam Repub Iran. 2019 (1 Oct);33:104. https://doi.org/10.47176/mjiri.33.104

\section{Introduction}

Clinical empathy is the physician's ability to understand the patient's perspectives and experiences and convey this understanding to the patient (1). This process makes the patient feel respected and valued by the physician. Clinical empathy has positive outcomes, including increased patient satisfaction (2-6). In this regard, Hojat et al. found a correlation coefficient of 0.93 between patient

Corresponding author: Dr Shoaleh Bigdeli, bigdeli.sh@iums.ac.ir

1. Department of Medical Education, Faculty of Medicine, Iran University of Medical Sciences, Tehran, Iran

2. Center for Educational Research in Medical Sciences (CERMS), Iran University of Medical Sciences, Tehran, Iran

3. Medical Education Research Center, Health Management and Safety Promotion Research Institute, Tabriz University of Medical Sciences, Tabriz, Iran satisfaction and his perception of the physician's empathy (7). Reynolds and Scott also reported a positive relationship between clinical empathy and the patient's responses, such as pain relief, regulation of breathing and pulse, and reduced stress and anxiety (8). After an empathetic visit to the physician, diabetic patients showed fewer complications (9), and patients with a common cold

$\uparrow$ What is "already known" in this topic:

- Decline in empathy, an increased suspicion, and a decline in altruism were reported by almost all the medical students.

- The first step to promote clinical empathy in medical students is to identify its challenges.

$\rightarrow$ What this article adds:

- This qualitative study explained the challenges of promoting clinical empathy skills among medical interns.

- Two main challenges extracted: Overt challenges, which are tangible and objective, and covert challenges, which affect empathy in medical students in an implied and intangible way. - Management of financial and human resources are required to resolve the challenges in promoting clinical empathy in medical students.

- Also, patients and interns should also receive training on the principles of effective doctor-patient interactions. 
experienced the shortest possible duration of illness (10) and patients ability for self-help and life management increased (11).

Clinical empathy also prevents occupational burnout and stress in physicians and helps their well-being as a potentially-contributing factor. There is a positive relationship between physicians' quality of life and health and their level of empathy. An empathic physician is happier at work, enjoys patient visits, and is clinically competent $(12,13)$.

The positive effects of clinical empathy have made different institutions emphasize the importance of promoting clinical empathy among medical students (1416). Despite this emphasis, studies have shown that empathy gradually declines in medical students during their academic education (17-19). A decline has also been observed in the empathy of medical students in Iran (20). A systematic review conducted by Neumann et al. also showed a decline in empathy during the period of medical training and residency in 17 out of the 18 studies reviewed (3). In a study by Billings et al. in the USA, a decline in empathy, an increased suspicion and a decline in altruism were reported by almost all the medical students during their years of academic training (21). Despite the importance of clinical empathy, studies suggest that medical education leads to a descent in empathy $(5,22)$. The first step to promote clinical empathy in medical students is to identify its challenges. Since empathy is a psychosocial phenomenon, it should be studied in a real environment with human complexities; however, most studies on empathy have had a quantitative and selfassessment approach, and very few studies have adopted a qualitative approach (23). The present qualitative study was conducted to explain the challenges in promoting clinical empathy skills among medical interns in order to yield a deeper understanding of the issue.

\section{Methods}

\section{Study type}

In this qualitative, conventional, content analysis, categories were extracted from the first-hand data obtained through data collection.

\section{Participants and research environment}

The study setting consisted of teaching hospitals affiliated to Iran and Ardabil universities of medical sciences. Medical interns were selected through purposive sampling, which continued until the saturation of the data (total of 14). Six clinical professors were also included in the study to complete the data and answer the questions resulting from the interviews with the interns. Inclusion criteria for interns was passing an externship program and for professors was having a minimum of one-year clinical teaching experience. The interns were selected from a diverse background in terms of the academic year, gender, and marital status. The clinical professors were also diverse in terms of specialty, years of work experience, and having a managerial position.

\section{Data collection}

Data were collected through individual and face to face semi-structured interviews. Arrangements were made with the participants about the time, place, and duration of the interviews in advance. Therefore, the interviews were conducted at a suitable time and place. The purpose of the study was explained to the participants before the start of the interview. We did not use any interviewing guides. Each interview began with a general open-ended question about the challenges in promoting empathy skills in medical students and continued by asking probing questions to help clarify the ambiguities in participants' responses. The mean duration of each interview was 45 minutes. All the interviews were conducted by one of the members of the research team (NA) who had prior knowledge about communication and empathy skills but tried not to allow her previous knowledge interfere with the interviews.

\section{Data analysis}

Data were analyzed according to the Graneheim and Lundman method (24). The interviews were transcribed immediately after completion and were reviewed several times to obtain a general understanding. The meaning units were then extracted and classified as condensed units. After the initial classification, the condensed units were reclassified and given a meaning label. The subcategories were then organized based on their similarities and differences and were given suitable names.

\section{Trustworthiness}

Member-check and the researcher's ongoing engagement were two ways of ensuring the acceptability of the data. The research team reviewed the data to check their objectivity and expressed their views about the extracted codes until reaching a consensus. For accurate data auditing, the themes, categories, sub-categories, primary codes, and interviews will be kept safe and secure six months after publishing the data. To ensure the replicability of the data, two external auditors were asked to analyze a sample of the interview content.

In addition, researchers with various specializations and different perspectives analyzed the data. To provide triangulation (e.g. space, investigator, interdisciplinary and data), participants from different specializations and academic and occupational positions were interviewed.

\section{Ethical considerations}

The research proposal was approved by the ethics committees of Iran and Ardebil University of Medical Sciences, and the researchers were introduced to university-affiliated hospitals. Before starting the interviews, the participants were briefed on the study objectives, means of publishing the data, confidentiality of the collected data, lack of personal, financial, psychological or social damage, and the possibility of withdrawing from the study at any stage. Finally, the informed written consent was secured. 


\section{Results}

The mean age of interns (ten women and four men) were 26.2 years that was 46 for the clinical professors (two women and four men). The mean duration of academic education was 6.5 years in the interns and the mean work history was 13.8 years in the clinical professors.

The two main emerged themes were "overt" and "covert" challenges."

\section{Overt challenges}

This theme involves two categories, including patientoriented challenges and deficiencies of the environment.

\subsection{Patient-oriented challenges}

The factors affecting lack of improved empathy skills in medical students include patients' distrust and humiliating behavior towards interns.

\subsubsection{Patients' distrust}

The patients' trust in medical interns affects the quality of patient-intern relationship and the interns' empathy with the patients. The participants acknowledged that patients do not trust interns or have less trust in them, and this issue affects the process of clinical empathy improvement in medical students. For example, one participant stated: "Usually, they (the patients) do not that trust me at first, since they notice that I am hesitant about prescribing medications, so they lose their trust in me and stop talking" (M4, year-7 intern, 26 years old).

The effect of the patients' trust on their comfort and ease of communication was described as: "The patient should be able to trust me so that he can talk to me with ease and make a relationship" (M11, year-7 intern, 25 years old). Another participant discussed patient distrust and said: "The patients were calmed down, because they trusted the resident, but they didn't trust me or listen to me" (M7, year-5 intern, 26 years old). The participants complained about the patients' inappropriate and insulting behaviors and considered them as empathy barriers. For example, one of the professors said: "In many cases, patients insult young doctors; 'you are nobody', 'you are not educated', 'I have no time', etc., which is too much annoying for young physicians and prevents building a good rapport" (M15, female professor, nephrology subspecialty, 20 years of work experience as department head, 50 years old).

Tolerating the patients' misconduct leaves no room for empathy by the interns. One participant explained: "We had a badly-behaving patient last night who yelled a lot, and if we were to yell as well, what would happen to the hospital? So we just had to put up with him" (M1, year-6 intern, 25 years old).

\subsection{Deficiencies of the environment}

The shortcomings and problems existing in clinical settings prevent the creation of ideal conditions for establishing a good rapport and empathy between interns and patients. This category contained two sub-categories, including ineffective physical environment and negative implications of the environment.

\subsubsection{Ineffective physical environment}

According to the participants, ineffective physical environment can be examined from two perspectives: Lack of conducive physical conditions for establishing a good rapport, and lack of recreational and sports facilities to remove and prevent occupational burnout in interns.

Lack of a private space to adhere to the patient's right of privacy can affect the quality of doctor-patient relationship. One participant argued: "Our clinic or hospital does not have the space required for the patients to have their own privacy. They cannot talk to us with ease, and this prevents communication" (M9, year-7 intern, 28 years old).

Another factor that indirectly prevents improved empathy in interns is occupational burnout in both interns and professors, and lack of resting facilities also worsens the situation. One participant said: "We have no recreational facilities -neither for the students nor for ourselves. We are just as tired as the students. We are crushed under this pressure" (M15, female nephrology subspecialty, 20 years of work experience as department head, 50 years old).

1.2.2. Negative implications of the environment

Analyzing participants' experiences showed that patients' dissatisfaction with the problems existing in the hospital prevents them from building a good relationship with the interns, and this issue affects the promotion of empathy among them. One participant explained: "The patients' dissatisfaction is mostly due to the system's problems, and they take it out on us, and don't build a good relationship with us" (M13, year-6 intern, 26 years old).

According to the participants, lack of conducive conditions in the environment was another challenge to promote empathy skills in interns. "The work conditions are so bad that I could see my colleagues' empathy gradually fade away, because the work environment and conditions and motivations become poorer day by day" (M17, female internal medicine professor, 15 years of work experience, 45 years old).

\section{Covert challenges}

Covert challenges affect students' clinical empathy and are not easily detectable. This category consists of two sub-categories, including role challenges in clinical training and the negative implications of the hidden curriculum.

\subsection{Role challenges in clinical training}

The role of trainees and interns during clinical training is a subject that has never been properly elaborated. This category includes two sub-categories, namely role confusion in the externship and internship periods.

\subsubsection{Role confusion in the externship period}

The participants frequently stated that, since in the externship period, they had been merely passive observers, the process of communicating with the patients has not properly formed in them. A participant explained: "We did not have to manage the patients that much during the externship period, and the program was mostly educational. Perhaps we took a brief history of them but did not know how to deal with or communicate with them" 
(M4, year-7 intern, 26 years old). Another participant said: "We were unable to communicate too much with the patients during the externship period, because the patients and their relatives were warned about us being students and did not give us much information" (M8, year-6 intern, 25 years old).

\subsubsection{Role confusion in the internship period}

According to the participants, the sudden delegation of responsibility to interns makes them mentally preoccupied and adversely affects their clinical empathy and communication. One participant commented: "Everybody has vain expectations, and think that once you have passed your pre-internship exams, you suddenly become a know-it-all, and this confuses us" (M12, year-6 intern, 25 years old). Another participant said: "We are under a lot of stress because of the responsibilities associated with having to treat a patient, and we try to ask questions and don't think about issues such as communication" (M14, year-7 intern, 25 years old).

\subsection{The implications of the hidden curriculum}

The implications of the hidden curriculum inadvertently and negatively affect students who have no preparation to attend clinical settings. This category has three subcategories, including the doctor-dominated culture, viewing patients as customers, and the humiliating behaviors of the professors.

\subsubsection{The doctor-dominated culture}

One of the concerns of the participants was the doctors' dominance over the patients. A participant described: "Our attendees convey to the patients that they have the first and last word, and that the patients have to listen to them and that they are in charge of treating the patients as they wish. If any patient is allowed to speak or talk about his previous symptoms and complications at all or not-it is totally up to the attending faculty member and not the patient" (M10, year-5 intern, 26 years old). Another participant argued: "No doctor likes the patients or their relatives to interfere in the treatment procedures. That is why I try to be firm and do not allow the patients or their relatives to interfere" (M9, year-7 intern, 28 years old).

2.2.2. Viewing the patients as customers (a commodity)

The attempt to attract more patients is a factor that imposes the professors' view of "patients as customers" on the interns, and conveying this attitude affects empathy in the interns. For example, one participant explained: "Right now, the argument is who has the most beds and the most income. This is the system's ailment, and students notice it "(M15, female professor, nephrology subspecialty, 20 years of work experience as department head, 50 years old). The professors' view of patients as a commodity that has to be attracted, affects the students' behaviors and actions. One participant said: "When we have too many patients, there is nothing in it for us, but they [the professors] get some money for each patient that they visit" (M1, year-6 intern, 25 years old).

\subsubsection{The humiliating conduct of professors}

According to the participants, professors who humiliate the students affect their feelings, and this, in turn, affects the promotion of clinical empathy skills in students. One participant said: "We have a professor who talks rudely in the morning report and insults the students and disheartens them" (M5, year-7 intern, 25 years old). Another participant stated: "Over these years, I have seen that some professors humiliate students, and students have been severely influenced by this conduct" (M3, year-6 intern, 25 years old). Insulting students in a crowd will surely have more damaging effects. One participant said: "There were students who had five times of internship in the same ward; however, they quit halfway through the semester because they were treated harshly by the attends in front of the public" (M6, year-7 intern, 34 years old).

\section{Discussion}

The present study was conducted to explain the challenges in promoting clinical empathy skills in medical students. The classification of the codes extracted from participants' interviews led to two main challenges: Overt challenges, which are tangible and objective, and covert challenges, which affect empathy in medical students in an implied and intangible way.

Patient-oriented challenges are overt. A patient is a constituent part of doctor-patient relationship. The way the patients communicate affects the quality of interns' communication and clinical empathy. According to the participants, the patients' trust and the quality of their attitude toward medical students are two important factors in this process.

The patient's trust in the doctors is defined as the patient's optimistic acceptance of his vulnerability and the belief that the doctor will take care of his concerns (25). Study conducted by Bronholm et al. (2017) showed that creating and maintaining trust is a key factor in doctorpatient relationships (26). Because of the imbalance between doctors and patients in terms of medical knowledge and position, doctor-patient relationship is largely dependent on doctor's experience and skills in solving patient's medical problems (26). The trust in doctor's capabilities leads to active engagement of patients in the process of treatment and medical decisionmaking (27). Therefore, doctors and interns need to treat patients safely and securely so that they can attract their trust (28).

The result indicated patients' humiliating behavior toward students as a challenging factor. In a study conducted by Billings (2011), the participants reported harassment by the patients (21). In a study by Ahmadipour and Vafadar 93\% medical students said that they had been verbally or physically threatened, and $83 \%$ of them said that they had been harassed by the patients' companions (29).

To resolve patient-oriented challenges and subsequently to promote empathy skills in medical students, clinical professors should familiarize the patients with interns and their responsibilities and brief them on the special position of interns. Clinical professors also need to teach communication and history-taking skills to their students, so that they can be better prepared for communicating with the patients. Moreover, patients also need to learn the principles of doctor-patient interactions through the 
media. Building a culture that respects doctors and their special position will inevitably affect clinical empathy.

The deficiencies of the environment are the other challenge to improve empathy in medical students. The results of a study conducted by Bigdeli et al. showed that students need improved clinical conditions and facilities (30) .Coping with stressful settings can lead to a decline in empathy (31) and the lack of physical facilities is another stressful factor.

The negative implications of the environment resulting from unfavorable clinical conditions and heavy workloads prevent the promotion of clinical empathy in interns. In a study conducted by Ahrweiler (2014), work pressure and time constraints negatively affected the promotion of empathy in doctors (32). In Tavakol et al. study, the participants proposed time constraints as a challenge that led to a decline in clinical empathy (15). The stress experienced in clinical settings was also discussed in a study by Afghani et al., and a large number of patients and time constraints were identified as stressful factors (33). The optimal provision and management of financial and human resources to improve patient care and ensure welfare of the clinical personnel can pave the way for improving empathy skills in medical students.

Role challenges in clinical training comprised another theme in this study. Although this theme has been less directly addressed in articles, it has been an implied theme in a study conducted by Henning (2011) that showed role transparency and clarification as priorities in clinical training according to year-4 interns (34). Early exposure periods are therefore recommended to be held for trainees before the externship period so that they can receive training on their roles and the expectations of them and can promote doctor-patient relationship skills.

Another challenge in the present study was the implications of the hidden curriculum, which has often been discussed in articles on empathy and professionalism (35). Billings et al. defined the hidden curriculum as the unwanted messages conveyed by the professors, personnel, and residents in clinical settings and training that exudes an aura of unprofessionalism to the students (21). Lempp defined hidden curriculum as a series of influences that act at the cultural and organizational level. In the studies reviewed by Jeffry, students discussed the absolute power inspired by this culture and stated that, since empathy has not been a priority of their professors, unlike biomedical knowledge and the technical aspects of treatment, they did not consider empathy seriously (6).

In the present study, the doctor-dominated culture and viewing patients as customers were sub-categories of the hidden curriculum. In Murakami et al. study, the great emphasis on medical sciences followed by a lack of attention to patients and patient-oriented care were identified as part of the hidden curriculum (36). In a study by Billings et al. respondents believed that their clinical professors did not exhibit humanistic attributes as providers of patient care, and attached greater importance to academic skills rather than humanistic skills and regarded emotions as a threat. They have also discussed doctors' view of patients as customers and their little consideration of humanistic attributes; in their study, almost all the students acknowledged that they had witnessed unprofessional behaviors during their years of education, including little empathy with the patients and reduced lack of humanism. As a result, students may think that humanistic attitudes are not part of their role as a doctor (21).

In a meta-ethnographic study, Jeffry found that there is a lack of adequate attention to the patients' humanistic aspects in clinical settings (6). With the rapid increase in scientific technology and the greater emphasis on biomedical aspects, there is some level of concern about the little attention paid to patients; Spiro even used the phrase "unseen and unheard patients" to refer to this subject. Medicine is currently faced with the phenomenon of "dehumanization", and the need for "re-humanization" is dire (37).

The professors' humiliating conduct comprised another hidden challenge in the present article.

In Mavis et al. study from 2000 to 2011, one-third of the students reported that they had been subjected to mistreatment, and clinical professors were identified as its main source (38). Intimidating questions, humiliation of the students, and offensive behaviors of the professors were the most common mistreatments reported by Scott et al. (39). In the study by Shoukat et al., more than half of the students complained about mistreatment by their professors and professors had a more significant role in mistreatment of students compared to other groups (40).

A large number of studies around the world have confirmed the harassment of medical students. The prevalence of mistreating students means that there is an urgent need for creating an educational environment directed toward learning clinical skills empathy emphasized (40). Discrepancies between the formal and the hidden curriculum challenged students with distressing emotional and ethical dilemmas (41). Hurtful and humiliating behaviors found to be the most hidden and the less obvious. As a cultural issue, solving the problem of mistreating students requires multidimensional and longterm measures and a commitment to providing a curriculum emphasizing professionalism (39).

In contrast, to unprofessional behaviors committed by professors, the formal curriculum stresses interdisciplinary practice, collegiality, and patient-centered care. Interpersonal tensions and eye-rolling can subvert formal talk of collegiality, and praise of interdisciplinary teamwork contrasts with the hierarchy of our institutions (42). These results suggest that professors are willing to teach professional behaviors such as empathy, but they are not aware that the best way to convey these values to students is through observation and their own possession of these behaviors. Professors can be asked to list the values that are important to them at the beginning of the course, to be more focused on them during the course.

With the shift toward patient care activities in clinical training, the hidden curriculum becomes more important than ever, and a tangible decline emerges in the students' empathy, especially at the beginning of the third year of medical studies. This finding shows the large gap between 
what we expect of medical students in the future and what they learn in practice today, and this gap is a significant barrier to the realization of the goals of medical education, which is to foster humanistic and empathetic doctors (43). According to students routinely recognize tension or misalignment between their prior idealized notions of medical practice, as often conveyed through the formal curriculum, and their actual experiences in clinical training (44), It is suggested that the gap between formal and hidden curricula is resolved. It is also recommended that students become familiar with the consequences of experiencing clinical settings before they enter these environments. As a strategy, teachers should be attracted to adhere to professional principles and ethical competence. And current professors should be retrained for attaining further clinical empathy in medical settings.

\section{Limitations}

This content analysis study includes its provision of indepth understanding of experiences elicited from a small group of participants. The findings of this study are limited to the experience of a group of medical students at two medical schools. For further understanding, qualitative studies, particularly those using ethnographic methods, involving students, doctors, patients, and their relatives should be pursued. It also recommended that in future studies to be interviewed exclusively by psychologists and psychiatrists may obtain different information.

\section{Conclusion}

Resolving the challenges in promoting clinical empathy in medical students requires not only to provide and manage the needed financial and human resources, but the patients and interns should also receive training on the principles of effective doctor-patient interactions. Furthermore, the professionalism of professors should be strengthened, and both the official and the hidden curricula should be revised and modified as the most effective strategies to promote empathy in medical students.

\section{Acknowledgements}

This study is part of a Ph.D. thesis in Medical Education. The research proposal was approved and funded by the Office of Continuing Education of the School of Medicine of Iran University of Medical Sciences with the approval No. 6, dated October 21, 2014. The agreement to conduct this research was registered at Ardabil University of Medical Sciences by the number 4373951 dated 29,11,2014. The authors wish to express their gratitude to all the participants, including clinical professors and medical interns. The authors are grateful to Dr. Fazlollah Ahmadi professor of Tarbiat Modares University (TMU) whose scholarly comments and consultations improved the content, Dr. Ladan Fata, associate professor of Iran University of Medical Sciences (IUMS) and Dr. Mehrisadat Mehdizadeh assistant professor of Mashhad University of Medical Sciences
(MUMS).

\section{Conflict of Interests}

The authors declare that they have no competing interests.

\section{References}

1. Youssef F, Nunes P, Sa B, Williams S. An exploration of changes in cognitive and emotional empathy among medical students in the Caribbean. IJME. 2014;5(1):185-92.

2. Hojat M, Vergare MJ, Maxwell K, Brainard B, Herrine S, Isenberg $\mathrm{GA}$, et al. The devil is in the third year: A longitudinal study of erosion of empathy in medical school. Acad Med. 2009;84:1182-91.

3. Neumann M, Edelhäuser F, Tauschel D. Empathy decline and its reasons :A systematic review of studies with medical students and residents. Acad Med. 2011;86(8):996-1009.

4. Rohani C, Sedaghati Kesbakhi M, J M. Clinical empathy with cancer patients: a content analysis of oncology nurses' perception. Patient Prefer Adherence. 2018;12:1089-98.

5. Ferreira-Valente A, Monteiro JS, Barbosa RM, Salgueira A, Costa P, Costa M. Clarifying changes in student empathy throughout medical school: a scoping review. Adv in Health Sci Educ J. 2017;22(5):1293313.

6. Jeffrey D. A meta-ethnography of interview-based qualitative research studies on medical students' views and experiences of empathy. Med Teach. 2016;38(12):1214-20.

7. Hojat M. Empathy in Health Professions Education and Patient Care. Switzerland: Springer; 2016.

8. Brunero S, Lamont S, Coates M. A review of empathy education in nursing. Nurs Inq. 2010;17:65-74.

9. Derksen F, Bensing J, Lagro-Janssen A. Effectiveness of empathy in general practice: a systematic review. $\mathrm{Br} \quad \mathrm{J}$ Gen Pract. 2013;63(606): $76-\mathrm{e} 84$

10. Rakel D, Barrett B, Zhang Z, Theresa Hoeft T, Chewningd B, Marchanda L, et al. Perception of empathy in the therapeutic encounter: Effects on the common cold. Patient Educ\&Couns. 2011;85(3):390-7.

11. Mercer SW, Neumann M, Wirtz M, Fitzpatrick B, Vojt G. General practitioner empathy, patient enablement, and patient-reported outcomes in primary care in an area of high socio-economic deprivation in Scotland-A pilot prospective study using structural equation modeling. Patient Educ Couns. 2008;73(2):240-5.

12. Paro H, Silveira P, Perotta B, Gannam S, Enns S, Giaxa R, et al. Empathy among medical students: Is there a relation with quality of life and burnout? Plos One. 2014;9(4).

13. Newton BW. Walking a fine line: is it possible to remain an empathic physician and have a hardened heart? Front Hum Neurosci 2013;7:233.

14. Berg K, Majdan J, Berg D. Medical students' self-reported empathy and simulated patients' assessments of student empathy: An analysis by gender and ethnicity. Acad Med. 2011;86:984-8.

15. Tavakol S, Dennick R, Tavakol M. Medical students' understanding of empathy: a phenomenological study. Med Educ. 2012;46:306-16.

16. Kataoka H, Koide N, Hojat M, Gonnella J. Measurement and correlates of empathy among female Japanese physicians. BMC Med Educ. 2012;12(48).

17. Aomatsu M, Otani T, Tanaka A, Ban N, Valen J. Medical students' and residents' conceptual structure of empathy: A qualitative study Educ Health. 2013;26(1):4-8.

18. Roff S. Reconsidering the "decline" of medical student empathy as reported in studies using the Jefferson Scale of Physician EmpathyStudent version (JSPE-S). Med Teach. 2015:1-4.

19. Newton BW, Barber L, Clardy J, Cleveland E, O’Sullivan P. Is there hardening of the heart during medical school? Acad Med. 2008;83(3).

20. Shariat V, Habibi M. Empathy in Iranian medical students: Measurement model of the Jefferson scale of empathy. Med Teach. 2013;e1-e6(Early Online).

21. Billings ME, Lazarus ME, Wenrich M, Curtis JR, Engelberg RA. The effect of the hidden curriculum on resident burnout and cynicism. J Grad Med Educ. 2011;3(4):503-10.

22. Nunes P, Williams S, Sa B, Stevenson K. A study of empathy decline in students from five health disciplines during their first year of training. Int J Med Edu. 2011;2:12-7. 
23. Gleichgerrcht E, Decety J. Empathy in clinical practice: how individual dispositions, gender, and experience moderate empathic concern, burnout, and emotional distress in physicians. Plos One. 2013;8( 4 ):e61526.

24. Graneheim UH, Lundman B. Qualitative content analysis in nursing research: concepts, procedures and measures to achieve trustworthiness. Nurse Educ Today. 2004;24(2):105-12.

25. Muller E, Zill J, Dirmaier J, Harter M, Scholl I. Assessment of trust in physician: a systematic review of measures. Plos One. 2014;9(9):e106844.

26. Broholm-Jorgensen M, Guassora AD, Reventlow S, Dalton SO, Tjornhoj-Thomsen T. Balancing trust and power: a qualitative study of GPs perceptions and strategies for retaining patients in preventive health checks. Scand J Prim Health Care. 2017;35(1):89-97.

27. Bernhardsson S, Larsson MEH, Johansson K, Oberg B. "In the physio we trust": A qualitative study on patients' preferences for physiotherapy. Physiother Theory Pract. 2017;33(7):535-49.

28. Holwerda N, Sanderman R, Pool G, Hinnen C, Langendijk JA, Bemelman WA, et al. Do patients trust their physician? The role of attachment style in the patient-physician relationship within one year after a cancer diagnosis. Acta Oncologica. 2013;52(1):110-7.

29. Ahmadipour H, Vafadar R. Treatment of medical students in clinical settings. strides in development of medical education. J Med. 2013;10(3):335-42.

30. Bigdeli Sh, Pakpour V, Aalaa M, Shekarabi R, Sanjari M, Haghani $\mathrm{H}$, et al. Clinical learning environments (actual and expected): perceptions of Iran University of Medical Sciences nursing students. Med J Islam Repub Iran. 2015;29(173).

31. Lopez L, Katz JT. Creating an ethical workplace:Reverberations of resident work hours reform. Acad Med. 2009;84(3):315-20.

32. Ahrweiler F, Neumann M, Goldblatt H, Hahn E, Scheffer C. Determinants of physician empathy during medical education: hypothetical conclusions from an exploratory qualitative survey of practicing physicians. BMC Medical Education. 2014;14:122.

33. Afghani B, Besimanto S, Amin A, Shapiro J. Medical students' perspectives on clinical empathy training. Educ Health. 2011;24(1)

34. Henning M, Shulruf B, Hawken S, Pinnock R. Changing the learning environment: the medical student voice. Clin Teach. 2011;8:83-7.

35. Pedersen R. Empathy development in medical education -A critical review. Med Teach. 2010;32:593-600.

36. Murakami M, Kawabata H, Maezawa M. The perception of the hidden curriculum on medical education: an exploratory study. Asia Pac Fam Med J. 2009;8(1):9.

37. Spiro H. The practice of empathy. Acad Med. 2009;84:1177-79.

38. Mavis B, Sousa A, Lipscomb W, Rappley MD. Learning about medical student mistreatment from responses to the medical school graduation questionnaire. Acad Med. 2014;89(5):709-11.

39. Scott KM, Caldwell P, Barnes EH, Barrett J. "Teaching by humiliation" and mistreatment of medical students in clinical rotations: a pilot study. Med J Aust. 2015;203(4):185-e6.

40. Shoukat S, Anis M, Kella DK, Qazi F, Samad F, Mir F, et al. Prevalence of mistreatment or belittlement among medical students A cross sectional survey at a private medical school in Karachi, Pakistan. Plos One. 2010;5(10).

41. Lamiani G, Leone D, Meyer E, Moja E. How Italian students learn to become physicians: A qualitative study of the hidden curriculum. Med Teach. 2011;33:989-96.

42. Mahood SC. Medical education: Beware the hidden curriculum. CFP. 2011;57(9):983-5.

43. Hendelman W, Byszewski A. Formation of medical student professional identity: categorizing lapses of professionalism, and the learning environment. BMC Med Educ. 2014;14:139-49.

44. Gaufberg EH, Batalden M, Sands R, Bell SK. The hidden curriculum: what can we learn from third-year medical student narrative reflections? Acad Med. 2010;85(11):1709-16. 